\title{
Continuous femoral nerve block for postoperative analgesia after knee rotationplasty for osteosarcoma of the distal femur
}

Hiroaki Murata, Mai Yoshizaki, Tetsuya Hara.

Nagasaki University School of Medicine, Department of Anesthesiology, Nagasaki, Japan.

\section{Background and Aims}

Osteosarcoma commonly affects the distal femur of adolescents. Knee rotationplasty is a limb salvage surgery alternative to above-knee amputation for osteosarcoma around the knee. Appropriate postoperative analgesia is important to facilitate rehabilitation.

\section{Case presentation}

- An 18-year-old male $(178 \mathrm{~cm}, 72 \mathrm{~kg})$ with osteosarcoma at the left distal femur was planned to undergo an excision of the tumor and subsequent rotationplasty.

- Anesthesia was maintained with propofol and remifentanil $(0.2 \mu \mathrm{g} / \mathrm{kg} / \mathrm{min})$ in combination with single-shots sciatic nerve block $(0.25 \%$ levobupivacaine $20 \mathrm{ml})$ and femoral nerve block $(0.25 \%$ levobupivacaine $20 \mathrm{ml})$.

- Osteosynthesis was performed between the remaining femur and tibia.

- Femoral, tibial and common peroneal nerves were isolated and preserved.

- Vessels were required resection and microsurgical anastomosis was required.

- Anesthesia time: 19 h 23 min, surgery time: 17 h 20 min, bleeding: $1238 \mathrm{ml}$ (no blood transfusion required)

- At the end of surgery, intravenous acetaminophen $1000 \mathrm{mg}$ and flurbiprofen axetil $50 \mathrm{mg}$ were administered and continuous femoral nerve block was initiated.

- At emergence, fentanyl $100 \mu \mathrm{g}$ was intravenously administered.

Figure 1: Pictures of left knee rotationplasty before surgery with planned excision lines $(A)$ and at the completion of surgery (B).

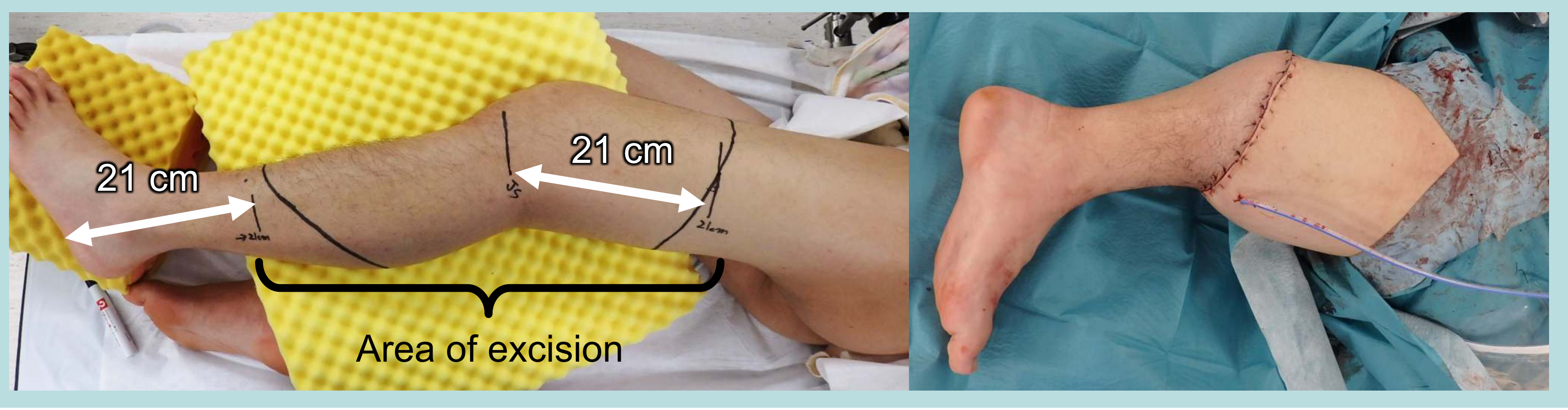

Figure 2: Postoperative course of the patient until postoperative day (POD) 4.

\begin{tabular}{|c|c|c|c|c|c|c|}
\hline $\begin{array}{c}\text { Postoperative } \\
\text { time [h] }\end{array}$ & 3 & 4 & 7 & 27 (POD2) & 51 (POD3) & 75 (POD4) \\
\hline $\begin{array}{c}\text { Wound pain } \\
\text { (NRS) }\end{array}$ & 0 & 0 & 0 & 2 & 2 & 1 \\
\hline Events & Oxygen off & Start oral fluid & Start regular diet & $\begin{array}{c}\text { Start self } \\
\text { postural change }\end{array}$ & $\begin{array}{c}\text { Removal of iv } \\
\text { route }\end{array}$ & $\begin{array}{c}\text { Removal of urine } \\
\text { catheter }\end{array}$ \\
\hline
\end{tabular}

continuous femoral nerve block

( $0.17 \%$ levobupivacaine $5 \mathrm{~mL} / \mathrm{h}$, no leakage and no catheter dislodgement)

Oral acetaminophen (400 mg every $6 \mathrm{~h}$ )

NRS, numerical rating scale $(0=$ no pain and $10=$ worst imaginable pain $)$

\section{Conclusions}

- Continuous femoral nerve block provided excellent analgesia after knee rotationplasty.

- Sparing postoperative opioids facilitated early oral intake.

- Preservation of contralateral lower extremity motor function also facilitated self postural change. 